\title{
Auto generation of the center of gravity of tubular structures during crush deformation
}

\begin{abstract}
Displacement of the center of gravity (COG) of tubular structures with various polygonal cross-sections is numerically investigated under an axial crush using the program code of ANSYS/LS-DYNA. A subroutine is developed using this code to calculate the COG of the deformed shape, during and after the crush. The effect of wall thickness on displacement of the COG is also investigated. Displacement of the COG decreases as the number of edges increases; it is a reasonable symmetric-deformed shape for the number of edges beyond eight. An even number of edges leads to a more symmetric displacement of the COG. The effect of the number of polygonal edges on symmetric deformation of the COG becomes more prominent as the initial wall thickness decreases. The higher number of edges stabilizes the deformed shape and the value of the mass moment of inertia of the deformed shape about the $y$ axis (Iyy). The value of the mass moment of inertia about the x-z axes (Ixz) in comparison with I yy can be neglected in the case of dealing with an axial crush along the y direction.
\end{abstract}

Keyword: ANSYS/LS-DYNA; Center of gravity; Finite element; Tubular structure 